\title{
Waveform Agile Sensing Approach for Tracking Benchmark in the Presence of ECM using IMMPDAF
}

\author{
Gnane Swarnadh SATAPATHI, Srihari PATHIPATI
}

Dept. of Electronics and Communication Engineering, National Institute of Technology Karnataka, Mangalore, 575025 Karnataka, India

ec13f02@nitk.edu.in,srihari@nitk.ac.in

Submitted July 28, 2016 / Accepted November 28, 2016

\begin{abstract}
This paper presents an efficient approach based on waveform agile sensing, to enhance the performance of benchmark target tracking in the presence of strong interference. The waveform agile sensing library consists of different waveforms such as linear frequency modulation (LFM), Gaussian frequency modulation (GFM) and stepped frequency modulation (SFM) waveforms. Improved performance is accomplished through a waveform agile sensing technique. In this method, the selection of waveform to be transmitted at each scan is determined, by jointly computing ambiguity function of waveform and Cramer-Rao Lower Bound (CRLB) matrix of measurement errors. Electronic counter measures (ECM) comprises of stand-off jammer (SOJ) and self-screening jammer (SSJ). Interacting multiple model probability data association filter (IMMPDAF) is employed for tracking benchmark trajectories. Experimental results demonstrate that, waveform agile sensing approach require only 39.98 percent lower mean average power compared to earlier studies. Further, it is observed that the position and velocity root mean square error values are decreasing as the number of waveforms are increasing from 5 to 50.
\end{abstract}

\section{Keywords}

Clutter, electronic countermeasures, root mean square error, target tracking

\section{Introduction}

The chief objective of target tracking is to increase the probability of detection (i.e. to detect and track true targets) and reduce false alarms. The presence of strong interference sources (ECM, clutter, false alarm (FA) and multipath) significantly degrade the performance of radar target tracking. SSJ and SOJ are regarded as effective ECM techniques. In SOJ, the enemy aircrafts are present outside the surveillance region of the radar. It radiates high power jamming signals into the radar main lobe or side lobe, to misguide the enemy target, which is entering into the surveillance region.
In SSJ, the jammer itself is present on the enemy target and sends erroneous signals to the radar. The main objective of both these ECM techniques is to corrupt radar measurements, and hence deceive the radar to track false targets. In addition to ECM, clutter and multipath in the environment collectively increases the complexity of radar to detect and track the true targets. Therefore, there is a strong need to adapt radar parameters to get improved observations, which will increase the performance of tracking in the presence of strong interference.

Adaptive waveform selection is considered as an important electronic counter counter measure (ECCM) for tracking targets in the presence of ECM [1]. Varying waveforms from scan to scan can give significant information about true targets and helps to locate them accurately by improving radar measurements. The main objective here, is to enhance the tracking performance by building waveform library with multiple waveforms and select the waveform to be transmitted based on prediction of next state of the target.

Benchmark problem with six standard target trajectories has been proposed in [2]. Interacting Multiple Model (IMM) adaptive estimator was presented as an efficient target tracking algorithm for maneuvering targets [3]. Significant further research was conducted on tracking benchmark trajectories in the presence of ECM [4-7]. In all these, SOJ and range gate pull off (RGPO) were viewed as major ECM techniques. An important contribution for benchmark target trajectories has been reported [1], [8] and applied IMMPDAF as a track filter for all six benchmark trajectories in the presence of ECM. A number of potential research problems that have to be carried out in future were recommended in [8]. Significant among them are adaptive waveform selection using various radar waveforms, tracking closely spaced targets, considering on-board jammer, including background clutter and incorporating multipath effects.

Alternatively, interacting multiple model/ multiple hypothesis tracker (IMM/ MHT) solution to the radar benchmark problem was proposed in [9], which requires less radar resources than [8]. But IMM/MHT was computationally complex when it was applied to practical situations. 
Benchmark problem with IMMPDAF was further extended by incorporating background clutter with fixed LFM waveform [10]. A new set of algorithms for radar management were introduced in [11], [12], which presented post detection integration techniques for benchmark problem to jointly reduce radar energy and to improve accuracy of target measurements in the presence of ECM. In all the above proposed algorithms, only single LFM waveform was used for benchmark tracking.

Waveform agile sensing algorithms were developed to select a particular waveform from a bank of waveforms, which aims to maximize the probability of detection and minimize the mean square error. A novel approach was recommended using adaptive waveform selection for linear target tracking using Kalman filter in clutter free environment and was extended to include clutter [13], [14]. Different optimization techniques for waveform design were explored in [15-17]. Improved version of tracking non-linear model by using dynamic waveform selection was suggested in [18]. Generalized frequency modulated waveforms for non-linear scenario were presented in [19], [20]. Both these approaches yielded better results for various scenarios. Adaptive waveform selection was recommended for multi static radars using IMMPDAF model in [21].

This paper presents waveform agile sensing based approach to track benchmark trajectories in the presence of strong interference. Current work focuses on improving tracking performance by constructing a bank of radar waveforms (LFM, GFM and SFM) up to 50, including SSJ, SOJ, false alarm and incorporating multipath effects with background clutter.

The paper is organized as follows. Section 2 presents problem formulation. Waveform agile sensing for neutralizing ECM and clutter is developed in Sec. 3. Section 4 discusses IMMPDAF algorithm. Simulation results and discussion are included in Sec. 5. Conclusion and future work has been incorporated in Sec. 6 .

\section{Problem Formulation}

In the proposed waveform agile sensing approach, a phased array radar $(10 \mathrm{GHz}$ operating frequency and a rectangular array with 900 elements) with minimum variance distortion less response (MVDR) adaptive beamformer has been used to obtain the measurements. The transmit waveform is selected based on CRLB of scan-to-scan measurement errors from a bank of frequency coded radar waveforms (LFM, GFM and SFM) up to 50. A point target is assumed and closely spaced targets are ignored. Cell Averaging-Constant False Alarm Rate (CA-CFAR) adaptive thresholding technique is employed. Various electronic counter measures are included such as SOJ and SSJ. In addition, background clutter, multipath effects and false alarms are also incorporated. IMMPDAF is applied to track all the six benchmark trajectories in this complex scenario. The work flow of entire simulation process is briefly illustrated in Fig. 1.

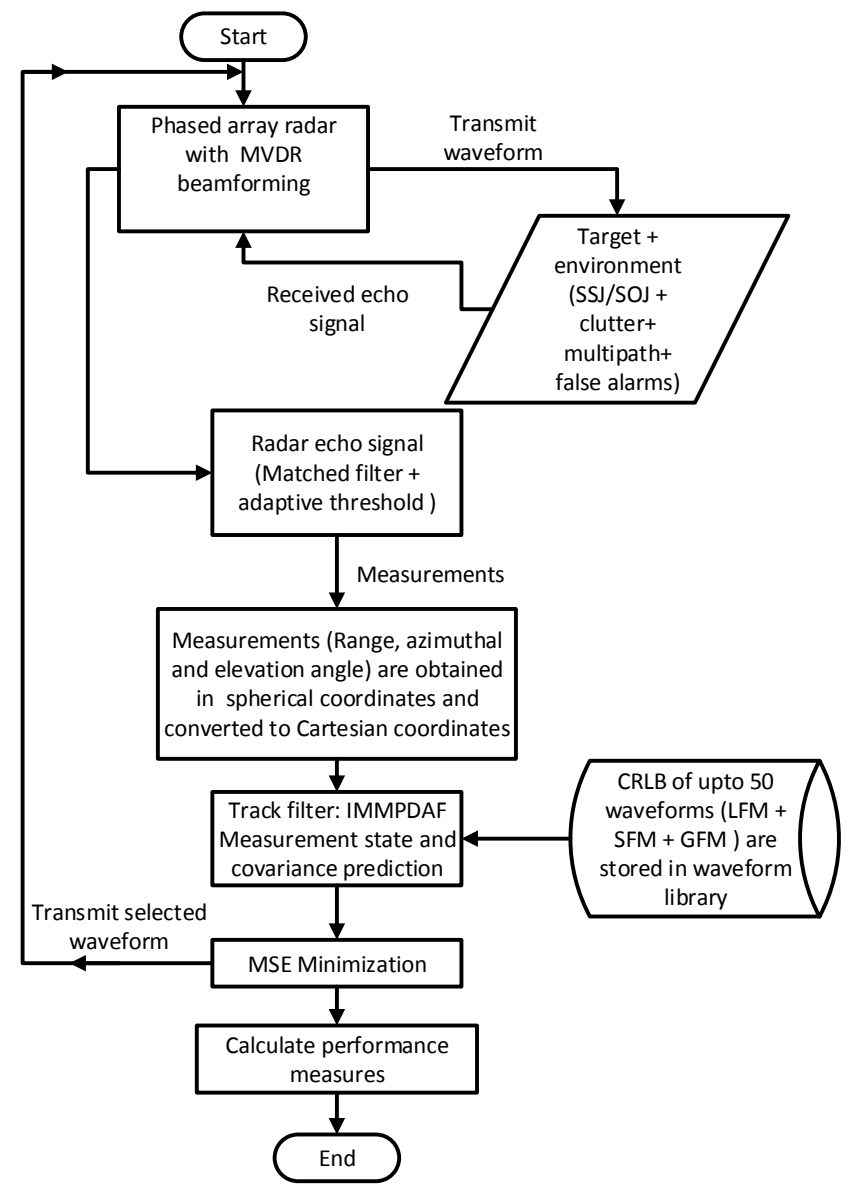

Fig. 1. Flowchart of entire simulation process.

\subsection{Measurement Model}

The measurements received from the radar are in spherical coordinates (range, azimuthal angle and elevation angle). Phased array radar scans the entire region in both azimuth and elevation direction simultaneously. The measurements from spherical coordinates are converted in to Cartesian coordinates with reference to radar position. The measurements from the radar is given by

$$
Z_{i}=\left[Z_{i x}, Z_{i y}, Z_{i z}\right]
$$

where $Z_{i x}, Z_{i y}$ and $Z_{i z}$ are the positions in $x, y \& z$ directions respectively of $i^{\text {th }}$ element of the measurement vector with respect to origin of the radar. The environment consists combination of clutter, ECM, false alarm and multipath. Hence, the resultant measurements obtained from the radar is

$$
Z=Z_{\text {target }}+Z_{\text {clutter }}+Z_{\text {jammer }}+Z_{\text {false alarm }}+Z_{\text {multipath }}
$$

where $Z_{\text {target }}, Z_{\text {clutter }}, Z_{\text {jammer }}, Z_{\text {false alarm, and }} Z_{\text {multipath }}$ are measurements due to target, clutter, jamming, false alarm and multipath at the radar receiver respectively.

\subsection{Jammers}

The main task of jammer is to hamper the radar functionality [22]. Jammers send high power radio frequency signals to fool the radar throughout its operating bandwidth. 
These jammers can be on-board or with an escort to the enemy target. Generally there are two types of jammers namely self-screening jammer (SSJ) and stand-off jammer (SOJ). The noise generated by jammer is measured in terms of effective radiated power (ERP) and is formulated as

$$
\mathrm{ERP}=\frac{G_{\mathrm{J}} P_{\mathrm{J}}}{L_{\mathrm{J}}}
$$

where $G_{\mathrm{J}}$ is gain in jammer antenna, $P_{\mathrm{J}}$ is jammer transmitted power and $L_{\mathrm{J}}$ is total loss in jammer. The following subsections describe briefly about SSJ and SOJ.

\subsubsection{Self-Screening Jammer}

Self screening jammers (SSJs) are well known as selfprotection jammers and are positioned on-board the target. They take advantage in the vicinity of radar surveillance area and send noise echoes to the radar main beam so as to crack the lock of radar. Signal to Jamming ratio $(\mathrm{S} / \mathrm{J})$ for self screening jammer case [22] is given as

$$
\frac{S}{J}=\frac{P_{\mathrm{t}} \tau G \sigma B_{\mathrm{J}}}{\mathrm{ERP} \cdot 4 \pi R^{2} L}
$$

where $P_{\mathrm{t}}$ is peak transmit power, $G$ is antenna gain, $\tau$ is radar pulse width, $\sigma$ is radar cross section, $B_{\mathrm{J}}$ is Jammer bandwidth, $R$ is range, $B_{\mathrm{r}}$ is receiver bandwidth, $L$ is receiver loss and ERP represents effective radiated power. Generally, it is considered that jammer power is greater than signal power transmitted by the radar i.e. $S / J<1$. Yet, when the target approaches radar, at a certain range signal power of radar will be equal to jamming power and this range is called as cross-over range. Beyond this cross-over range the jammer power is ineffective and is given by

$$
\left(R_{\mathrm{co}}\right)_{\mathrm{SSJ}}=\left[\frac{P_{\mathrm{t}} G \sigma B_{\mathrm{J}}}{4 \pi B_{\mathrm{r}} L \cdot \mathrm{ERP}}\right]^{1 / 2} .
$$

\subsubsection{Stand-Off Jammer}

Stand off jammers (SOJs) transmits noise signals from lethal range of the radar so as not to detect the enemy targets that are entering into the radar surveillance area. Signal to Jamming ratio for stand-off jammer is given by

$$
\frac{S}{J}=\frac{P_{\mathrm{t}} \tau G^{2} R_{\mathrm{J}}^{2} \sigma B_{\mathrm{J}}}{4 \pi \cdot \mathrm{ERP} \cdot R^{4} L}
$$

and cross-over range where jamming power is equal to signal power is

$$
\left(R_{\mathrm{co}}\right)_{\mathrm{SOJ}}=\left[\frac{P_{\mathrm{t}} G^{2} R_{\mathrm{J}}^{2} \sigma B_{\mathrm{J}} G_{\mathrm{pc}}}{4 \pi \cdot \mathrm{ERP} \cdot G^{\prime} B_{r} L}\right]^{1 / 4}
$$

where $R_{\mathrm{J}}$ is range of jammer from the radar and $G_{\mathrm{pc}}$ represents time bandwidth product. The target has to stay beyond this $\left(R_{\mathrm{co}}\right)_{\text {SOJ }}$ range, and try to send spurious signals to the radar for improper detection of the targets.

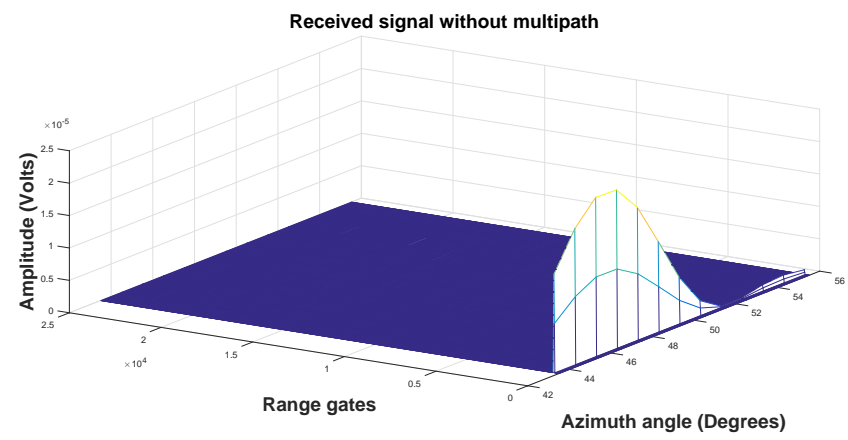

(a) Received signal without multipath Received signal with multipath

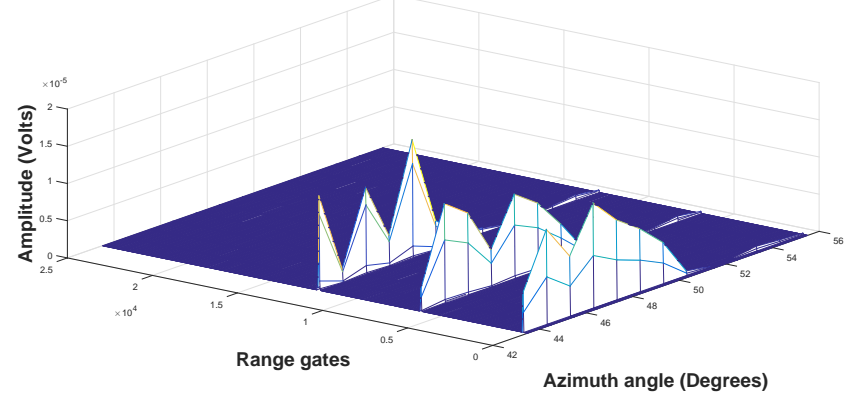

(b) Received signal with multipath.

Fig. 2. Received signal with and without multipath.

\subsection{Clutter Model}

Let $Z_{k, 1}, Z_{k, 2} \ldots Z_{k, n}$ be the measurements obtained from radar for a particular instant of time $k$. These measurements consists of false alarms with true measurements of the target due to the presence of clutter. If $\rho$ is considered as clutter density and $V_{k}$ as validation gate volume then false alarms are assumed to be Poisson distributed with mean $\rho V_{k}$. The observations from radar, which will fall in the validation region are only considered for tracking. Poisson probability for attaining $n$ false alarms is

$$
\mu(n)=\frac{e^{-\rho V_{k}}\left(\rho V_{k}\right)^{n}}{n !} .
$$

It is assumed that clutter is distributed uniformly in volume $V_{k}$.

\subsection{Multipath}

The echoes from the target return to the receiver other than direct path is known as multipath. Multipath generates false targets to appear and misleads the radar receiver. These multipath creates false targets, which are very difficult to distinguish from actual targets. Figure $2 \mathrm{a}$ depicts a received signal without multipath and Fig. 2 b shows two false targets are appearing due to multipath. If the echo is reflected from the rough surface, then error occurs in both azimuth and elevation angles due to diffuse scattering. Further, if the echo is reflected from building or non-flat land then error occurs significantly in azimuth angle. The major problem in tracking targets due to multipath effect is that, the false targets and actual targets seems to be coherent. The envelop sum of 
signals that are received at the receiver is considered to be Rayleigh distributed. The signals arriving at the receiver may have destructive or constructive interference. Let $R_{n}$ and $\phi_{n}$ be electric field and relative phase of $N$ multipath signals. Then, the total electric field at the receiver is given by

$$
\tilde{R}=\sum_{n=1}^{N} R_{n} \mathrm{e}^{j \phi_{n}} .
$$

It is assumed that $R_{n}$ and $\phi_{n}$ are independent and distributed uniformly. The probability density function of Rayleigh distribution is given by

$$
f_{\mathrm{R}}(r)=\frac{r}{\sigma^{2}} \mathrm{e}^{-\frac{r^{2}}{2 \sigma^{2}}} .
$$

Equation (10) is for slow fading and is valid $\forall r \geq 0$.

\subsection{Performance Measures}

In the target tracking literature, various performance measures have been proposed [23]. The following performance metrics (Tab. 1 in Appendix) are considered for evaluating the track performance of benchmark targets in the presence of ECM, clutter, multipath and false alarms.

\section{Neutralizing Techniques for ECM, Clutter and Multipath Effects}

In this section several mechanisms to neutralize ECM, clutter and multipath effects have been described. Waveform agile sensing, adaptive beamforming and adaptive thresholding techniques are successfully applied to neutralize these undesirable effects. The following subsections briefly describe these methods.

\subsection{Waveform Agile Sensing}

The main objective of adaptive waveform selection is to minimize tracking mean square error. Waveform selection process is based on signal to noise ratio, signal to clutter ratio and type of tracking algorithm applied. The parameter $\left(\Omega_{j}\right)$ of the waveform is selected so as to minimize the tracking MSE and is given by

$$
J\left(\Omega_{j}\right)=E_{X_{j}, Z_{j} \mid Z_{1: j}}\left[\left(X_{j}-\hat{X}_{j}\right)^{T}\left(X_{j}-\hat{X}_{j}\right)\right]
$$

where $E($.$) is the expectation operation over predicted and$ original state of measurements. $\hat{X}_{j}$ is the state estimate of $X_{j}$ for $j$ observations. Equation (11) is regarded as the cost function and aims to select the waveform parameter which yields minimum MSE at a particular scan $j$.

Single dynamic model may not exactly represent the motion of the target, as targets may maneuver. For this reason three PDAF filters are fused to IMM algorithm. Each filter is subjected for a particular target motion and will be running in parallel. The updated state estimate and covariance is the weighted combination of each individual PDAF state estimate and covariance respectively. The comprehensive derivation of IMM filter is done in [24].
The mean square error of specific track can be reduced by selecting waveform adaptively and this is accomplished by reducing trace of the updated error covariance matrix. The updated error covariance matrix is given by

$$
\begin{aligned}
P_{j+1 \mid j+1}\left(\Omega_{j+1}\right)= & P_{j+1 \mid j}-\left[1-\beta_{j+1}^{0}\right] W_{j+1}\left(\Omega_{j+1}\right) \\
& S_{j+1}\left(\Omega_{j+1}\right) W_{j+1}^{T}\left(\Omega_{j+1}\right)+\tilde{P}_{j+1}\left(\Omega_{j+1}\right) .
\end{aligned}
$$

From (12), it is evident that observation error covariance is a function of waveform parameter $\Omega_{j+1}$. Thus, the updated error covariance is also a function of $\Omega_{j+1}$. Since each PDAF in IMMPDAF is independently depended on $\Omega_{j+1}$, then the updated error covariance $P_{j+1 \mid j+1}^{\mathrm{IMM}}$ is a function of $\Omega_{j+1}$. The waveform library contains multiple waveforms with different combinations of parameters.

$$
\Omega_{j+1}=\min \left(\operatorname{Trace}\left(P_{j+1 \mid j+1}^{i *}\right)\right)
$$

where $i^{*}=\arg \max _{i} \mu_{j+1 \mid j+1}^{i}$.

The cost function for selecting waveform is briefly described in [13]. This is computed by evaluating the trace of updated covariance matrix for Kalman filter. The same procedure was extended to IMM filter in [21], [25] and (13) is chosen as one of the cost function. The waveform which gives highest mode probability of $i^{\text {th }}$ model of the filter and minimum covariance value is chosen for next scan. This technique has been well applied with various waveforms (LFM, GFM and SFM) to neutralize the undesirable effects.

\subsection{Adaptive Beamforming}

The beamforming aims to focus the main beam of radar in the intended direction and fix a null in the undesired direction. As the targets in practical scenario will not be stationery and the signal may arrive in any direction. Hence, conventional beamforming finds little use in these situations. The array weights needs to adapt continuously with change in environment and this process is known as adaptive beamforming. In the proposed approach, minimum variance distortion less response (MVDR) has been used to adapt weights of the beamformer. MVDR beamformer requires only direction of arrival (does not require any other information) to determine the beamformer weight vector

$$
W=\frac{R^{-1} S(\theta)}{S(\theta)^{H} R S(\theta)}
$$

where $S(\theta)$ is steering vector corresponding to a specific direction and $R$ is spatial covariance matrix. The key functionality of MVDR beamformer is to minimize the total output noise power by setting the gain as unity in a particular direction and this is done by adapting the weight vector of beamformer.

Figure 3 a shows the received signal with clutter, multipath and jamming before adaptive beamforming. It is very difficult to distinguish the target as it is embedded in clutter, ECM, multipath and false alarms. Figure $3 \mathrm{~b}$ shows 


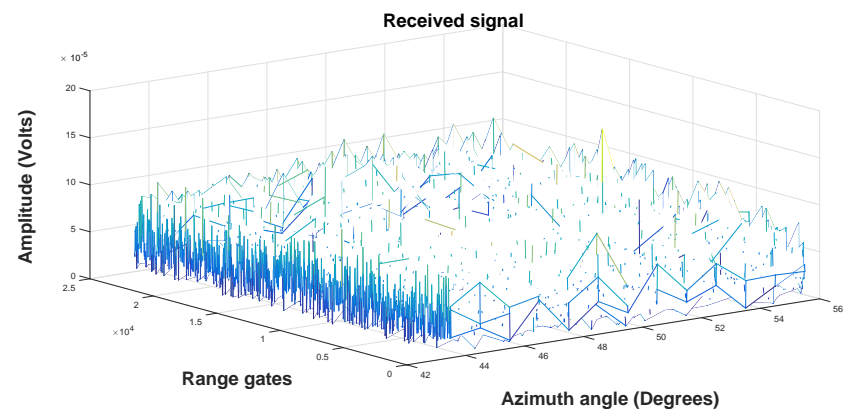

(a) Received signal before adaptive beamforming.

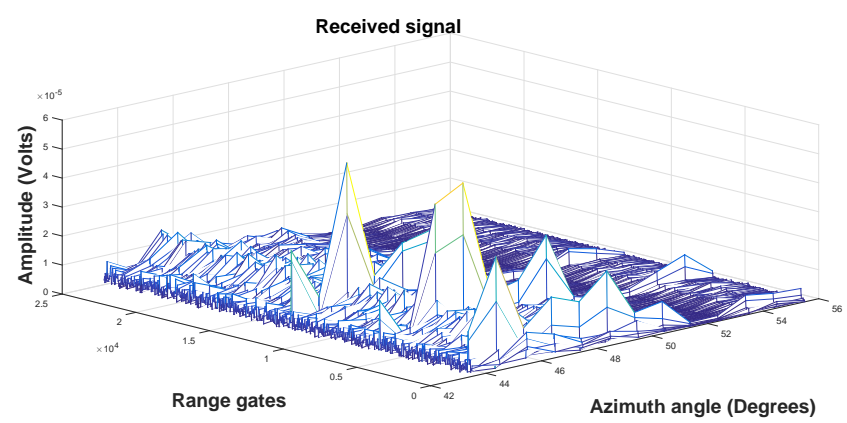

(b) Received signal after adaptive beamforming.

Fig. 3. Received signal before and after adaptive beamforming.

the received signal with adaptive beam forming. It can be visualized clearly that the noise is suppressed and targets can be identified along with multipath. MVDR beamformer is employed to suppress the ECM, clutter and false alarms in undesired direction.

\subsection{Adaptive Thresholding}

This subsection briefly describes about adaptive thresholding technique employed as a counter measure to ECM, clutter and multipath effects. Generally the received radar echo signal is compared to a fixed threshold value and hence declared the presence of a target based on, weather the matched filter output exceeds the threshold or not. In order to apply a fixed threshold in Neyman-Pearson detector, it seeks complete statistical information of the received echo signal. But, in practical situations, the statistical information of the received echo signal (corrupted with ECM, clutter and multipath effects) changes rapidly due to the change in environmental conditions. Therefore, there is a need for adaptive thresholding which varies according to change in environment and hence counter these unwanted effects.

The main function of the radar detector is to maximize the probability of detection and minimize the probability of false alarms. CA-CFAR is chosen for adaptive thresholding. In CA-CFAR, the cell under detection is known as cell under test (CUT). The neighboring cells of CUT are used to estimate the noise power. Some of the neighboring cells (lagging and leading) of CUT are referred as guard cells. These cells are ignored while estimating noise to avoid signal energy leakage from the CUT into training cells. The noise is estimated from the training cells which can be represented as

$$
\begin{aligned}
& U_{1}=\frac{1}{N_{1}} \sum_{i=1}^{N_{1}} x_{i} \\
& U_{2}=\frac{1}{N_{2}} \sum_{j=1}^{N_{2}} x_{j}
\end{aligned}
$$

where $N_{1}$ and $N_{2}$ represents the number of training cells, $x_{i}$ and $x_{j}$ denote the samples in each training cell respectively. Both $U_{1}$ and $U_{2}$ are combined to estimate the total background noise $P_{n}$. The noise estimate is multiplied with relevant scaling factor $\alpha$, so as to maintain probability of false alarm as constant. The detection threshold $T$ is given as

$$
T=\alpha P_{n}
$$

Threshold $(T)$ is compared with CUT in order to decide the presence of a target. Figure 4 shows detection of targets using adaptive thresholding. It clearly depicts that the target is detected along with multipath and few false alarms. CACFAR is applied to suppress ECM, clutter, false alarms and multipath effects.

The echo waveform from the target is corrupted by ECM, clutter, false alarms and multipath effects. Combination of the above effective techniques such as; Waveform agile sensing, adaptive beamformer and adaptive thresholding have been successfully applied to minimize the effects of these interferences.

\section{Interacting Multiple Model Proba- bility Data Association Filter}

This section provides the brief outline of the IMMPDAF track filter. The integration of interacting multiple model (IMM) with probability data association (PDA) makes an effective tracker for tracking maneuvering targets in the presence of ECM and false alarms [8].

\subsection{Probability Data Association Filter (PDAF)}

The PDA filter calculates the associated probability for each measurement based on validating a particular target. An assumption is made such that $m$ observations are validated for a particular scan. The equations required to update state and covariance for PDAF are given below:

Prediction of the state and measurement is given by

$$
\begin{gathered}
\hat{x}_{j+1 \mid j}=F \hat{x}_{j \mid j}, \\
\hat{z}_{j+1 \mid j}=H \hat{x}_{j+1 \mid j} .
\end{gathered}
$$

Covariance of the predicted state

$$
P_{j+1 \mid j}=F P_{j \mid j} F^{\prime}+Q
$$

Covariance with respect to measurement

$$
S_{j+1}\left(\Omega_{j+1}\right)=H P_{j+1 \mid j} H^{\prime}+C_{j+1}\left(\Omega_{j+1}\right)
$$




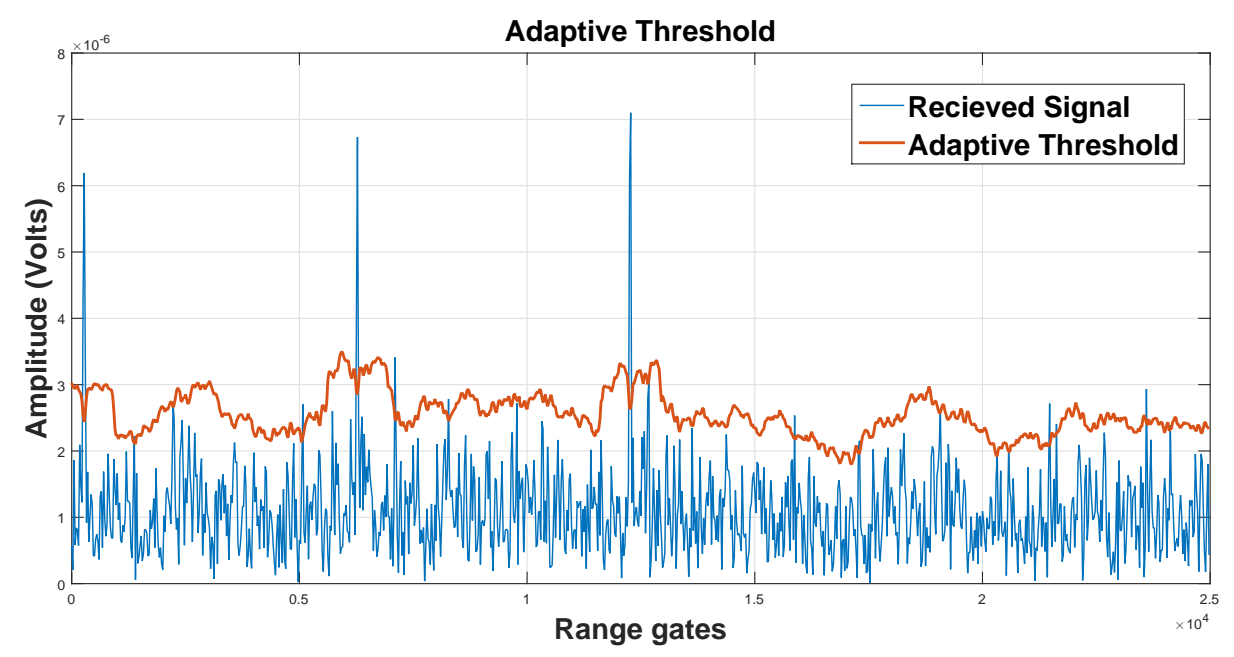

Fig. 4. Illustrating adaptive thresholding mechanism.

where $C_{j+1}$ is the observation error covariance matrix corresponding to $\Omega_{j+1}$ waveform. The validation region (gate)-the ellipsoid can be written as

$$
\mathcal{V}_{j+1}=\left\{z:\left[z-\hat{z}_{j+1 \mid j}\right]^{T} S_{j+1}\left(\Omega_{j+1}\right)^{-1}\left[z-\hat{z}_{j+1 \mid j}\right] \leq \gamma\right\}
$$

where $\gamma$ is the gate threshold determined by the chosen gate probability $P_{\mathrm{G}}$. Innovation corresponding to the $i$-th validated measurement

$$
v_{j+1}^{i}=z_{j+1}^{i}-\hat{z}_{j+1 \mid j} \quad i=1, \ldots, m_{j+1} .
$$

Volume of the validation region is given as

$$
V_{j+1}\left(\Omega_{j+1}\right)=c_{n_{z}}\left|\gamma S_{j+1}\left(\Omega_{j+1}\right)\right|^{1 / 2}
$$

where $c_{n_{z}}$ represents unit hypersphere volume with dimension $n_{z}$ (i.e. $\left.\left[c_{1}, c_{2}, c_{3}\right]=[2, \pi, 4 \pi / 3]\right)$. Probability of the $i$-th validated measurement is

$$
\beta_{j+1}^{i}\left(\Omega_{j+1}\right)= \begin{cases}\frac{e^{i}\left(\Omega_{j+1}\right)}{b+\sum_{l=1}^{m(j+1)} \mathrm{e}_{l}\left(\Omega_{j+1}\right)}, & i=1, \ldots, m(j+1), \\ \frac{b}{b+\sum_{l=1}^{m(j+1)} \mathrm{e}_{l}\left(\Omega_{j+1}\right)}, & i=0,\end{cases}
$$

$\beta_{0}(k+1)$ is association probability which represents that none of the measurement is correct

$$
\begin{gathered}
\mathrm{e}^{i}\left(\Omega_{j+1}\right) \triangleq \mathrm{e}^{-\frac{1}{2} v_{j+1}^{i} S_{j+1}\left(\Omega_{j+1}\right)^{-1} v_{j+1}^{i}}, \\
b \triangleq\left(\frac{2 \pi}{\gamma}\right)^{\frac{n_{z}}{2}} m(j+1) c_{n_{z}}^{-1} \frac{1-P_{D} P_{G}}{P_{D}} .
\end{gathered}
$$

State update

$$
\hat{x}_{j+1 \mid j+1}\left(\Omega_{j+1}\right)=\hat{x}_{j+1 \mid j}+W_{j+1}\left(\Omega_{j+1}\right) v_{j+1}\left(\Omega_{j+1}\right)
$$

where $W_{j+1}\left(\Omega_{j+1}\right)$ is filter gain and $v_{j+1}\left(\Omega_{j+1}\right)$ is known as combined innovation which is calculated as

$$
v_{j+1} \triangleq \sum_{i=1}^{m(j+1)} \beta_{j+1}^{i} v_{j+1}^{i}
$$

$$
W_{j+1}\left(\Omega_{j+1}\right) \triangleq P_{j+1 \mid j} H^{\prime} S_{j+1}\left(\Omega_{j+1}\right)
$$

Covariance associated to update state is given as

$$
\begin{aligned}
P_{j+1 \mid j+1}\left(\Omega_{j+1}\right)= & P_{j+1 \mid j}-\left[1-\beta_{j+1}^{0}\right] W_{j+1}\left(\Omega_{j+1}\right) \\
& S_{j+1}\left(\Omega_{j+1}\right) W_{j+1}^{T}\left(\Omega_{j+1}\right)+\tilde{P}_{j+1}\left(\Omega_{j+1}\right),
\end{aligned}
$$

$$
\begin{aligned}
\tilde{P}_{j+1}\left(\Omega_{j+1}\right) \triangleq & {\left[\sum_{i=1}^{m(j+1)} \beta_{j+1}^{i}\left(\Omega_{j+1}\right) v_{j+1}^{i} v_{j+1}^{i}{ }^{T}-v_{j+1} v_{j+1}^{T}\right] } \\
& \times W_{k+1}^{T}\left(\Omega_{j+1}\right) .
\end{aligned}
$$

Equations (27) and (29) represent the final update equation of state and covariance of PDAF respectively.

\subsection{Interacting Multiple Model Estimator (IMM)}

This subsection presents an outline of IMM method. Single targets can effectively be tracked by probability data association filter (PDAF) following a linear trajectory. But in practical scenarios, the targets may have higher acceleration turns, yielding abrupt maneuvers. Thus, there is a need to apply IMM technique, where PDAF filters are functioning in parallel and each PDAF filter having corresponding system matrix. The updated state estimate and covariance is the weighted sum of individual PDAF filters. Comprehensive explanation and derivation on IMM is given in [24]. In IMM, the state estimate is calculated by using different filters that run in parallel with the weighted combination of previous updated state estimates or with initial condition. 
Updated state estimate $\hat{x}_{j / j}^{l}$, updated error covariance $\hat{P}_{j / j}^{l}$ and model probability value $\mu_{j / j}^{l}$ of individual filter information is present for updating next iteration $j+1$ with observation value $z_{j+1}$. A concise derivation of IMM algorithm is given below:

- Computing mixed input to tracking filter

Predicted model probability is calculated by

$$
\mu_{j+1 \mid j}^{r}=\sum_{l=1}^{n} p_{l r} \mu_{j \mid j}^{l}
$$

where the model probability conditioned on $j$ is

$$
\mu_{j \mid j}^{l \mid r}=\left(1 / \mu_{j+1 \mid j}^{r}\right) p_{l r} \mu_{j \mid j}^{l}
$$

The mixed state estimate and covariance which is given as input to the PDAF filter is calculated by

$$
\begin{gathered}
\hat{x}_{j \mid j}^{0 r}=\sum_{l}^{n} \mu_{j \mid j}^{l \mid r} \hat{x}_{j \mid j}^{l}, \\
P_{j \mid j}^{0 r}=\sum_{l=1}^{n} \mu_{j \mid j}^{l \mid r}\left\{P_{j \mid j}^{l}+\left[\hat{x}_{j \mid j}^{l}-\hat{x}_{j \mid j}^{0 r}\right]\left[\hat{x}_{j \mid j}^{l}-\hat{x}_{j \mid j}^{0 r}\right]^{T}\right\} .
\end{gathered}
$$

- Updating mixed state estimate and covariance

The state estimate and covariance of each $r^{\text {th }}$ filter is updated from the input (32a) and (32b) to obtain updated state estimate $\left(\hat{x}_{j+1 \mid j+1}^{r}\right)$ and covariance $\left(\hat{P}_{j+1 \mid j+1}^{r}\right)$.

- Calculating model likelihood function

$$
\Lambda_{j+1}^{m}=\frac{1}{\sqrt{\left|2 \pi S_{j+1}^{r}\right|}} \mathrm{e}^{-\frac{1}{2}\left[\tilde{z}_{j+1}\right]^{T}\left[S_{j+1}^{r}\right]^{-1}\left[\tilde{z}_{j+1}\right]} .
$$

- Updating model probability of each filter

$$
\mu_{j+1 \mid j+1}^{r}=\frac{1}{b} \mu_{j+1 \mid j}^{r} \Lambda_{j+1}^{r}
$$

where $b$ is normalization factor

$$
b=\sum_{l=1}^{r} \mu_{j+1 \mid j}^{l} \Lambda_{j+1}^{l} .
$$

- Combining state estimate

$$
\hat{x}_{j+1 \mid j+1}^{\mathrm{IMM}}=\sum_{r=1}^{n} \mu_{j+1 \mid j+1}^{r} \hat{x}_{j+1 \mid j+1}^{r}
$$

$$
\begin{aligned}
P_{j+1 \mid j+1}^{\mathrm{IMM}}= & \sum_{r=1}^{n} \mu_{j+1 \mid j+1}^{r} \\
& \times\left\{P_{j+1 \mid j+1}^{r}+\left[\hat{x}_{j+1 \mid j+1}^{r}-\hat{x}_{j+1 \mid j+1}^{\mathrm{IMM}}\right]\right. \\
& {\left.\left[\hat{x}_{j+1 \mid j+1}^{r}-\hat{x}_{j+1 \mid j+1}^{\mathrm{IMM}}\right]^{T}\right\} . }
\end{aligned}
$$

Equations (36a) and (36b) represent the final update equations for state and covariance of IMM estimator. At any instant of time, any one of the state dynamic model in the parallel filters will be equal to the target trajectory and it gets automatically switched to it by evaluating model probability.

\section{Simulation Results}

This section deals with the simulation results and discussion. The following subsections describe six benchmark trajectories used in simulations and experimental results.

\subsection{Benchmark Trajectories}

Six benchmark trajectories from [8] have been used for testing waveform agile sensing in combined jamming (SOJ and SSJ), clutter and multipath scenario by applying IMMPDAF. Each trajectory turn rates, trajectory simulated time, constant velocity and target type is explained below:

\subsubsection{Benchmark Trajectory-1}

Initially the target is at position $[75,30,1.26] \mathrm{km}$ from radar and finally it reaches $[73.54,4.7,1.26] \mathrm{km}$ with $2 g$ and $3 g$ turns. The trajectory is simulated for $165 \mathrm{~s}$. The constant speed limit is maintained at $290 \mathrm{~m} / \mathrm{s}$. The trajectory shows large aircraft.

\subsubsection{Benchmark Trajectory-2}

Benchmark trajectory-2 is taken as second target trajectory for comparison of tracking performance with adaptive waveform selection. Initially the target is at a position $[47,-45,4.57] \mathrm{km}$ from the radar and finally reaches to $[34,-36.54,3.760] \mathrm{km}$ with $2.5 g$ and $4 g$ turns. Trajectory is simulated for $150 \mathrm{~s}$. The trajectory shows small maneuverable commercial jet. The true and estimated trajectory of benchmark-2 is shown in Fig. 5.

\subsubsection{Benchmark Trajectory-3}

The target makes $4 g$ acceleration turns with $45^{\circ}$ and $90^{\circ}$ at first $30 \mathrm{~s}$ and $60 \mathrm{~s}$ respectively. The maximum and minimum speed it travels is $457 \mathrm{~m} / \mathrm{s}$ and $274 \mathrm{~m} / \mathrm{s}$ respectively. The trajectory shows medium bomber. The simulation time of the trajectory is $145 \mathrm{~s}$.

\subsubsection{Benchmark Trajectory-4}

Target makes turn $45^{\circ}$ with $4 g$ and $6 g$ acceleration with a minimum speed of $251 \mathrm{~m} / \mathrm{s}$. The simulation time of trajectory is $184 \mathrm{~s}$ with maneuvering density of $9.92 \%$. The trajectory shows medium bomber. The true and estimated trajectory of benchmark-4 is shown in Fig. 6.

\subsubsection{Benchmark Trajectory-5}

The target takes complex manuvers with $5 g, 6 g$ and $7 g$ acceleration turns. The trajectory shows fighter aircraft. The simulation time of trajectory is $182 \mathrm{~s}$ with maneuvering density of $17.5 \%$.

\subsubsection{Benchmark Trajectory-6}

The target makes two $6 g$ and two $7 g$ turns and maintains a constant speed of $426 \mathrm{~m} / \mathrm{s}$. The trajectory shows fighter aircraft. The simulation time of trajectory is $188 \mathrm{~s}$ with maneuvering density of $18 \%$. The trajectory shows medium bomber. 


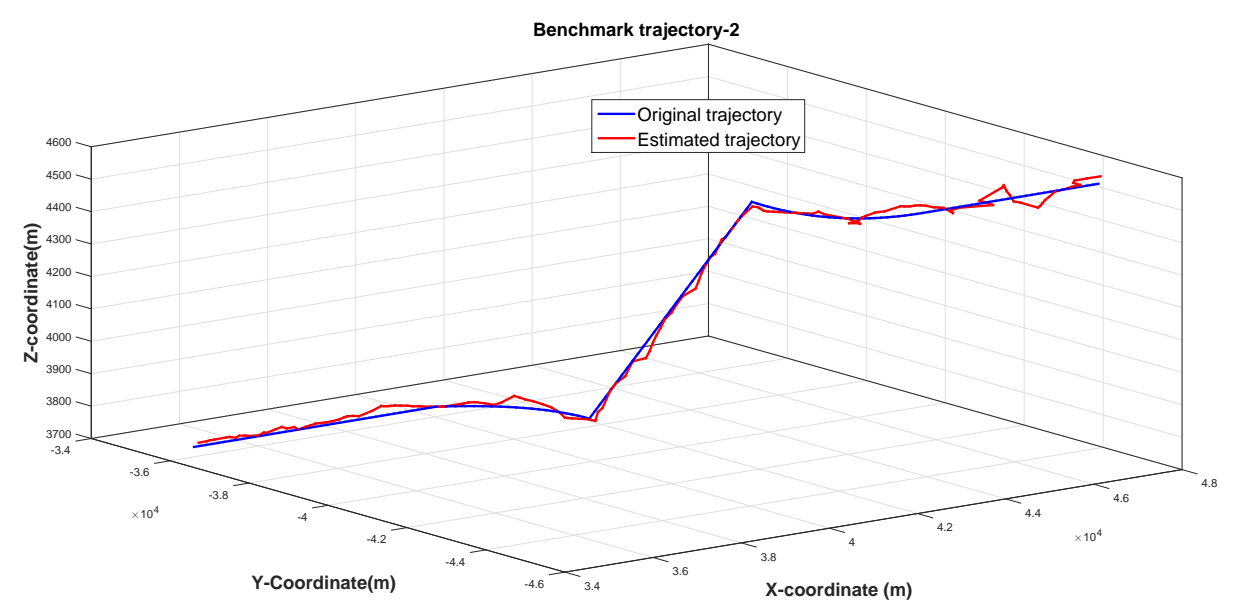

Fig. 5. Tracking of Benchmark target trajectory-2 in the presence of FA, SOJ, clutter and multipath.

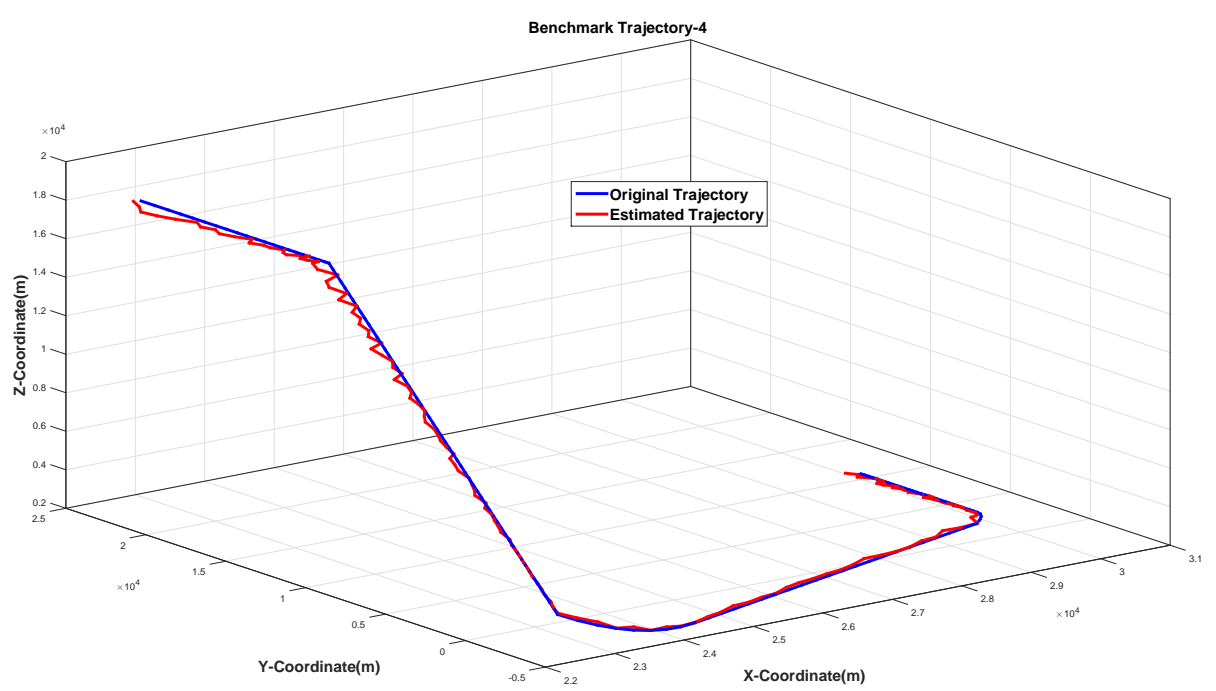

Fig. 6. Tracking of Benchmark target trajectory-4 in the presence of FA, SOJ, clutter and multipath.

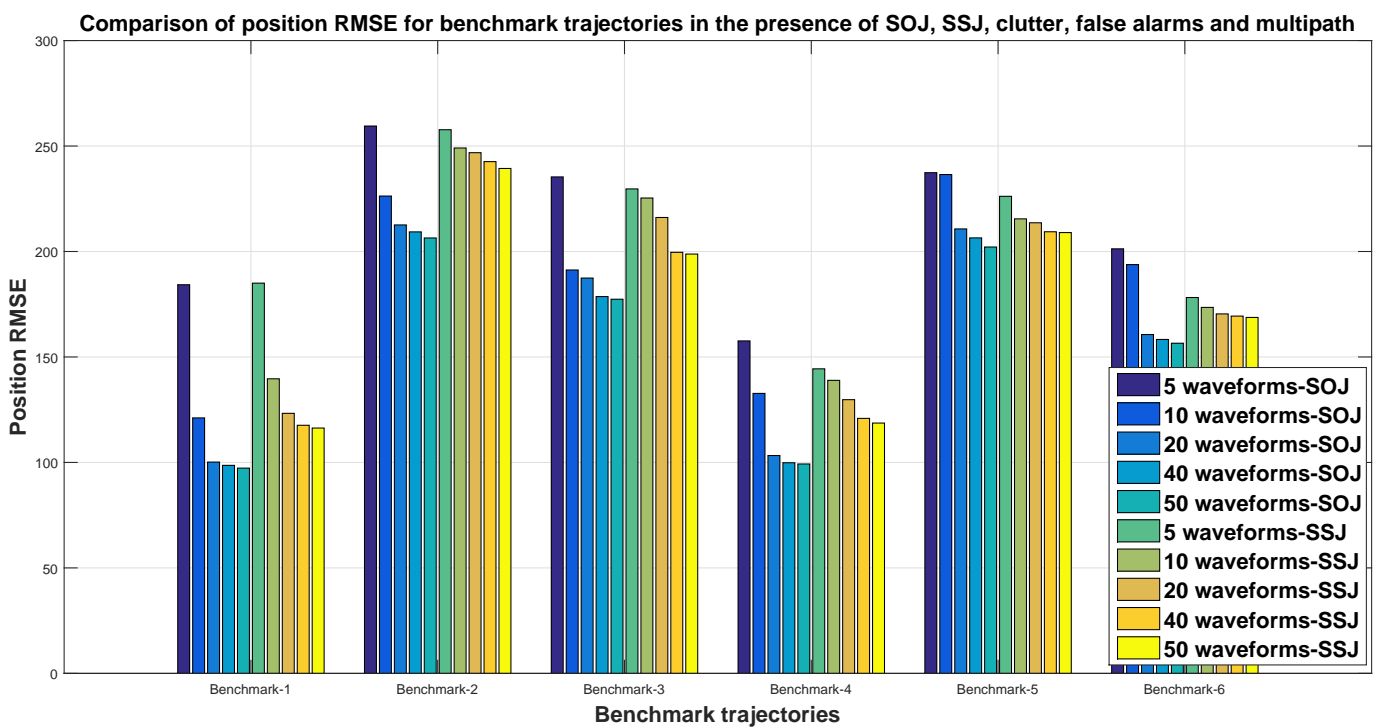

Fig. 7. Comparison of position RMSE for benchmark trajectories in the presence of SOJ, SSJ, clutter, false alarms and multipath. 


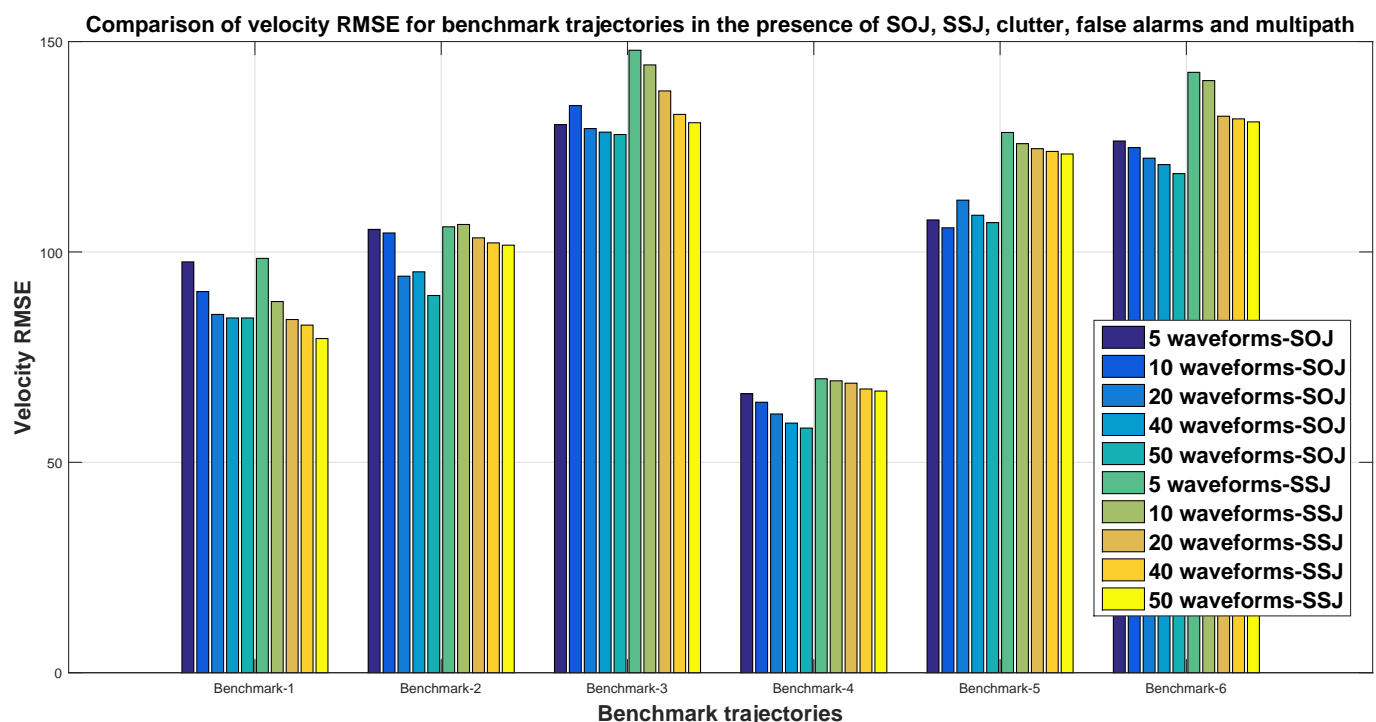

Fig. 8. Comparison of velocity RMSE for benchmark trajectories in the presence of SOJ, SSJ, clutter, false alarms and multipath.

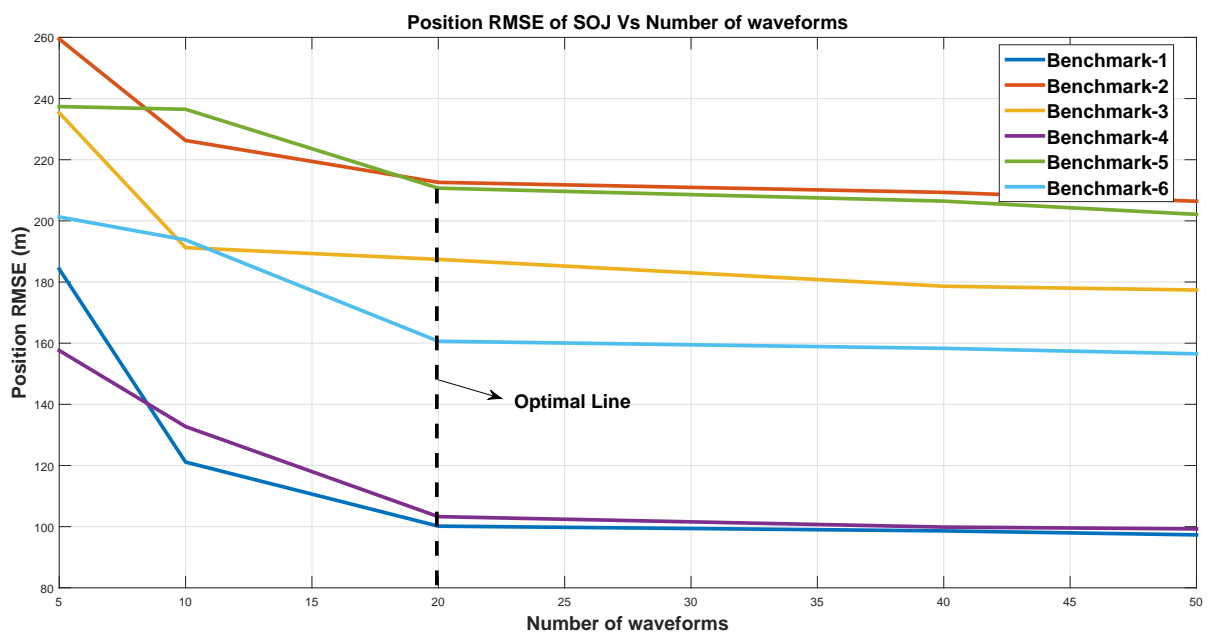

Fig. 9. Performance of position RMSE Vs number of waveforms for Benchmark Trajectories in the presence of SOJ, clutter, false alarm and multipath.

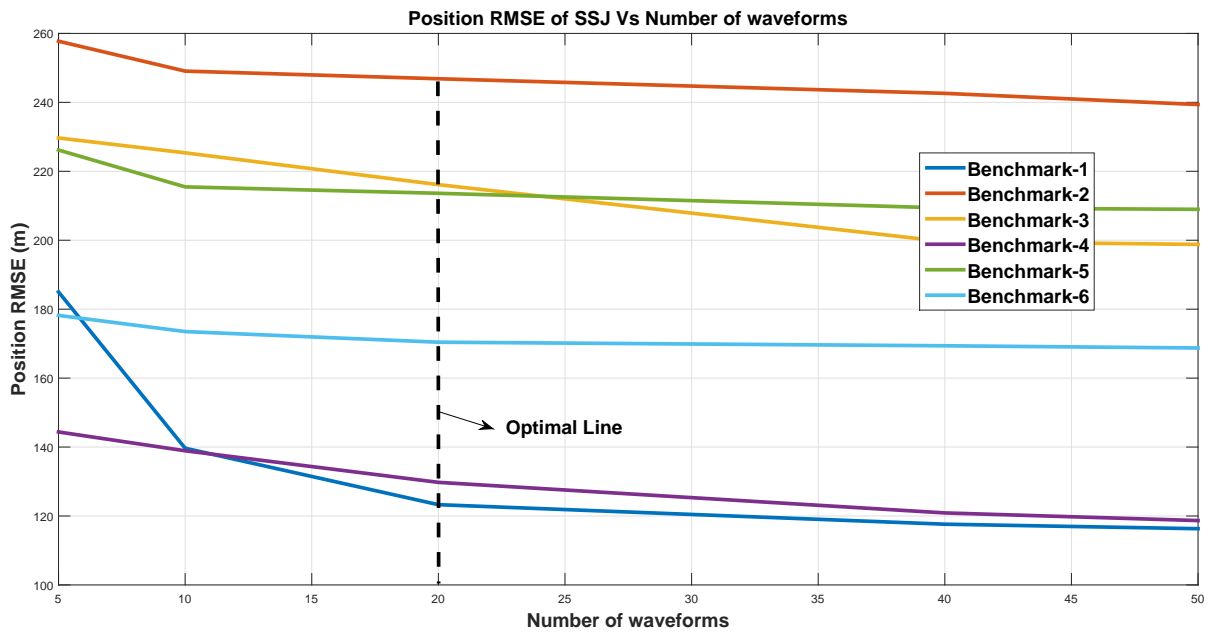

Fig. 10. Performance of position RMSE Vs number of waveforms for Benchmark Trajectories in the presence of SSJ, clutter, false alarm and multipath. 


\subsection{Simulation Results and Discussion}

Results for the above six benchmark trajectories with complex scenarios have been tabulated in Tab. 2 and Tab. 3 . Table 2 shows results in the presence of SOJ, clutter, multipath and FA are compared with earlier research study [10], which ignored multipath effects. On the other hand, results in the presence of SSJ, clutter, multipath and FA are tabulated in Tab. 3. Jammer on board the target (SSJ) have not been considered by any other earlier studies conducted, therefore these values have not been compared with any other results.

The tabulated results in Tab. 2 demonstrate that average power in current research study is significantly reduced for all benchmark trajectories except for benchmark-4. The simulation results reveals that proposed waveform agile sensing technique requires only $39.98 \%$ lower mean average power compared to past studies [10]. Besides that, the cost function $C_{1}$, which is associated with radar energy is significantly lowered in the current method. However, the cost function $C_{2}$, which is associated with radar time is little higher. The track loss performance is zero for benchmark-1, 2 trajectories and is $1.6 \%, 0.8 \%, 1.1 \%$ and $1.94 \%$ for benchmark-3, 4,5 and 6 trajectories respectively. From Tab. 2, it is also observed that position and velocity root mean square errors are higher. This clearly indicates that the multipath effect increased the measurement error along with the background clutter.

Furthermore, as the number of waveforms are increased from 5 to 50, both position RMSE and velocity RMSE values are decreasing (same is illustrated in Figures 7 to 8) [26]. It can also be observed that position RMSE with 50 waveforms in the current study is less than previous work for benchmark1 and for other benchmark trajectories its mean difference is $52.124 \mathrm{~m}$ for 50 waveforms. From Tab. 2, velocity RMSE in current study is higher than previous work with mean difference of $40.56 \mathrm{~m} / \mathrm{s}$. The earlier studies [10] ignored multipath in the environment. In the current research study multipath is incorporated in addition to the clutter and jamming, thus presenting a strong interference in the environment. Hence, the performance of velocity RMSE is slightly degraded when compared to previous studies.

The performance evaluation of IMMPDAF in the presence of SSJ, clutter, multipath and false alarm is tabulated in Tab. 3. It indicates the position and velocity RMSE values are decreasing as the number of waveforms increased for all benchmark trajectories. The average power for benchmark $-1,2$ and 6 are lower than benchmark-3, 4 and 5 trajectories. Track loss for benchmark 1, 2 and 5 is zero, whereas average track loss for benchmark 3,4 and 6 is $1.86 \%$. The $C_{1}$ and $C_{2}$ cost functions are lower for benchmark 1,2 and 6 compared to benchmark 3, 4 and 5 trajectories.

Plots are obtained by varying the number of waveforms in adaptive waveform selection with respect to position and velocity RMSE values for SOJ and SSJ scenarios. From Fig. 7 to 8, it is evident that the position and velocity RMSE of SSJ is significantly higher than SOJ for all benchmark trajectories. Further, as the number of waveforms in waveform library is increasing from 5 to 50 , the position and velocity RMSE values are decreasing.

Performance of position RMSE with number of waveforms considered for SOJ and SSJ cases are shown in Fig. 9 and 10 respectively. Optimal number of waveforms for both the cases are iluustrated with a dotted line as shown in Figures 9 and 10. In case of SOJ, the optimal RMSE observed for benchmark 1, 2, 4, 5 and 6 is at 20 waveforms. The optimal number of waveforms to be considered is 10 for benchmark- 3 in case of SOJ scenario. On the other hand, in case of SSJ, the optimal number of waveforms is widely varied for benchmark trajectories. For benchmark trajectory 2,5 and 6 the optimal number of waveforms to be considered is 10 in case of SSJ. Where as for benchmark trajectory 1 and 4 the optimality is achieved at 20 waveforms. For benchmark trajectory-3, the optimality is attained for 40 waveforms. Therefore, on an average the optimal performance is achieved at 20 waveforms for all such benchmarks in both the cases.

In this research work, closely spaced targets have not been considered and assumed only point targets. Besides, only frequency coded waveforms are used for waveform agile sensing. Further, ECM techniques such as; range gate pull off (RGPO) and velocity gate pull off (VGPO) are not examined. Future work can be carried out with these problem formulations.

The achieved results exhibits enhanced performance using waveform agile sensing approach in the presence of ECM, clutter, false alarm, and multipath effects. Hence, this method may be applied to track highly manuvering targets in the presence of strong interference.

\section{Conclusion}

Improved performance for tracking benchmark has been demonstrated using waveform agile sensing technique in the presence of strong interference. A number of frequency coded waveforms (LFM, SFM and GFM) up to 50 have been stacked in the waveform bank to enhance the track performance. IMMPDAF is applied to track benchmark with ECM (SOJ/SSJ), clutter and multipath effects. The simulation results show that proposed waveform agile sensing method requires only $39.98 \%$ lower mean average power compared to previous studies [10]. It is also evident that, as the number of waveforms are increasing from 5 to 50 , both position and velocity RMSE values are decreasing. Furthermore, position and velocity RMSE values are higher for SSJ than SOJ. This work can be further extended to incorporate phase coded waveforms to track benchmark. To conclude, potential research problems to be carried in future enlisted by Blair [8], have been addressed here and achieved significant results.

\section{Acknowledgments}

This research work is supported by Young Scientist Research Grant (No.SB/FTP/ETA-110/2014) of Department of Science and Technology, Govt. of India. 


\section{References}

[1] KIRUBARAJAN,T., BAR-SHALOM, Y., BLAIR, W., et al. IMMPDAF for radar management and tracking benchmark with ECM. IEEE Transactions on Aerospace and Electronic Systems, 1998, vol. 34, no. 4, p. 1115-1134. DOI: 10.1109/7.722696

[2] BLAIR, W., WATSON, G., HOFFMAN, S. Benchmark problem for beam pointing control of phased array radar against maneuvering targets. In Proceedings of the IEEE American Control Conference. 1994, vol. 2, p. 2071-2075. DOI: 10.1109/ACC.1994.752441

[3] DAEIPOUR, E., BAR-SHALOM, Y., LI, X. Adaptive beam pointing control of a phased array radar using an imm estimator. In Proceedings of the IEEE American Control Conference. 1994, vol. 2, p. 2093-2097. DOI: 10.1109/ACC.1994.752445

[4] BLAIR, W., WATSON, G., GENTRY, G., et al. Benchmark problem for beam pointing control of phased array radar against maneuvering targets in the presence of ECM and false alarms. In Proceedings of the IEEE American Control Conference. 1995, vol. 4, p. 2601-2605. DOI: 10.1109/ACC.1995.532318

[5] KIRUBARAJAN, T., BAR-SHALOM, Y., DAEIPOUR, E. Adaptive beam pointing control of a phased array radar in the presence of ECM and false alarms using IMMPDAF. In Proceedings of the IEEE American Control Conference. 1995, vol. 4, p. 2616-2620. DOI: 10.1109/ACC.1995.532321

[6] SLOCUMB, B., WEST, P., SHIREY, T., et al. Tracking a maneuvering target in the presence of false returns and ECM using a variable state dimension Kalman filter. In Proceedings of the IEEE American Control Conference. 1995, vol. 4, p. 2611-2615. DOI: 10.1109/ACC.1995.532320

[7] RAGO, C., MAHRA, R. Target tracking in the presence of ECM: A filter design tool. In Proceedings of the IEEE Twenty-Ninth Southeastern Symposium on System Theory. 1997, p. 514-518. DOI: 10.1109/SSST.1997.581720

[8] BLAIR, W., WATSON, G., KIRUBARAJAN, T., et al. Benchmark for radar allocation and tracking in ECM. IEEE Transactions on Aerospace and Electronic Systems, 1998, vol. 34, no. 4, p. 1097-1114. DOI: 10.1109/7.722694

[9] BLACKMAN, S., DEMPSTER, R., BUSCH, M., et al. IMM/MHT solution to radar benchmark tracking problem. IEEE Transactions on Aerospace and Electronic Systems, 1999, vol. 35, no. 2, p. 730-738. DOI: $10.1109 / 7.766953$

[10] ANGElOVA, D., SEMERDJIEV, E., MIHAYLOVA, L., et al. An IMMPDAF solution to benchmark problem for tracking in clutter and standoff jammer. In Proceedings of the International Conference on EuroFusion. 1999, p. 123-128.

[11] BEHAR, V., ANGELOVA, D., VASSILEVA, B., et al. A set of algorithms for radar management and target tracking in the presence of SOJ. Comptes Rendus de l'Academie Bulgare des Sciences, 2001, vol. 54, no. 7, p. 17.

[12] BEHAR, V., KABAKCHIEV, C. Radar waveform allocation by using post detection integration. Comptes Rendus de l'Academie Bulgare des Sciences, 2002, vol. 55, no. 1, p. 63.

[13] KERSHAW, D. J., EVANS, R. J. Optimal waveform selection for tracking systems. IEEE Transactions on Information Theory, 1994, vol. 40, no. 5, p. 1536-1550. DOI: 10.1109/18.333866

[14] KERSHAW, D. J., EVANS, R. Waveform selective probabilistic data association. IEEE Transactions on Aerospace and Electronic Systems, 1997, vol. 33, no. 4, p. 1180-1188. DOI: 10.1109/7.625110

[15] RAGO, C., WILLETT, P., BAR-SHALOM, Y. Detection-tracking performance with combined waveforms. IEEE Transactions on Aerospace and Electronic Systems, 1998, vol. 34, no. 2, p. 612-624. DOI: $10.1109 / 7.670395$
[16] NIU, R., WILLETT, P., BAR-SHALOM, Y. Tracking considerations in selection of radar waveform for range and range-rate measurements. IEEE Transactions on Aerospace and Electronic Systems, 2002, vol. 38, no. 2, p. 467-487. DOI: 10.1109/TAES.2002.1008980

[17] HONG, S. M., EVANS, R. J., SHIN, H. S. Optimization of waveform and detection threshold for range and range-rate tracking in clutter. IEEE Transactions on Aerospace and Electronic Systems, 2005, vol. 41, no. 1, p. 17-33. DOI: 10.1109/TAES.2005.1413743

[18] SIRA, S. P., PAPANDREOU-SUPPAPPOLA, A., MORRELL, D. Characterization of waveform performance in clutter for dynamically configured sensor systems. In Proceedings of the Waveform Diversity and Design Conference. 2006.

[19] SIRA S. P., MORRELL, D. Dynamic configuration of time-varying waveforms for agile sensing and tracking in clutter. IEEE Transactions on Signal Processing, 2007, vol. 55, no. 7, p. 3207-3217. DOI: 10.1109/TSP.2007.894418

[20] SIRA S. P., LI, Y., PAPANDREOU-SUPPAPPOLA, A., et al. Waveform-agile sensing for tracking. IEEE Signal Processing Magazine, 2009, vol. 26, no. 1, p. 53-64. DOI: 10.1109/MSP.2008.930418

[21] NGUYEN, N., DOGANCAY, K., DAVIS, L. Adaptive waveform selection for multistatic target tracking. IEEE Transactions on Aerospace and Electronic Systems, 2015, vol. 51, no. 1, p. 688-701. DOI: 10.1109/TAES.2014.130723

[22] MAHAFZA, B. R. Radar Signal Analysis and Processing Using MATLAB. 1st ed. CRC Press, 2008. ISBN: 9781420066432

[23] GORJI, A. A., THARMARASA, R., KIRUBARAJAN, T. Performance measures for multiple target tracking problems. In Proceedings of the 14th IEEE International Conference on Information Fusion (FUSION). 2011, p. 1-8. ISBN: 978-1-4577-0267-9

[24] BAR-SHALOM, Y., LI, X. R., KIRUBARAJAN, T. Estimation with Applications to Tracking and Navigation: Theory Algorithms and Software. John Wiley \& Sons, 2004. ISBN: 978-0-471-41655-5

[25] SAVAGE, C. O., MORAN, B. Waveform selection for maneuvering targets within an IMM framework. IEEE Transactions on Aerospace and Electronic Systems, 2007, vol. 43, no. 3, p. 1205-1214. DOI: 10.1109/TAES.2007.4383612

[26] MIAO, L., ZHANG, J. J., CHAKRABARTI, C. et al. Algorithm and parallel implementation of particle filtering and its use in waveformagile sensing. Journal of Signal Processing Systems, 2011, vol. 65, no. 2, p. 211-227. DOI: 10.1007/s11265-011-0601-2

[27] BLAIR, W., WATSON, G., et al. Benchmark for Radar Resource Allocation and Tracking Targets in the Presence of ECM. Sep. 1996, 67 pages. [Online] Cited 2016-07-28. Available at: http://www.dtic.mil/dtic/tr/fulltext/u2/a286909.pdf

\section{About the Authors ...}

Gnane Swarnadh SATAPATHI (corresponding author) was born in Visakhapatnam, India. He is currently pursuing Ph.D. in National Institute of Technology Karnataka, Surathkal in the area of radar target tracking.

Srihari PATHIPATI was born in Nellore, India. He obtained masters degree in communication engineering and signal processing from University of Plymouth, UK and recieved his Ph.D. from Andhra University, India in 2012. He is currently working as Assistant Professor in National Institute of Technology Karnataka, Surathkal. His research interests include radar signal processing and target tracking. He is a senior member of IEEE. 


\section{Appendix:}

\begin{tabular}{|c|c|c|c|}
\hline Sl.No & $\begin{array}{l}\text { Performance } \\
\text { metrics }\end{array}$ & Description & Reference \\
\hline 1 & $\begin{array}{l}\text { Root Mean } \\
\text { Square Error } \\
\text { (RMSE) }\end{array}$ & $\begin{array}{l}\text { It measures the difference between actual value and } \\
\text { estimated value. } \\
\qquad \text { RMSE }=\sqrt{\frac{1}{K} \sum_{i=1}^{k}\left(x_{i}-\hat{x_{i}}\right)^{2}} \\
\text { where } x_{i}=\text { Actual value; } \hat{x_{i}}=\text { Predicted value; } K=\text { No } \\
\text { of observations. }\end{array}$ & [23] \\
\hline 2 & Track Loss & $\begin{array}{l}\text { The track is declared to be lost if the error in the } \\
\text { estimated value of the target is greater than } 1.5 \text { range } \\
\text { gates in range. It measures the percentage of tracks } \\
\text { that are lost during simulation. }\end{array}$ & [8], [23] \\
\hline 3 & $\begin{array}{l}\text { Cost functions } \\
\left(C_{1} \& C_{2}\right)\end{array}$ & $\begin{array}{l}C_{1} \text { : It corresponds to period of operation when radar } \\
\text { energy is critical. } \\
\qquad \begin{array}{r}C_{1}=\bar{E}_{\text {ave }}+10^{3} \bar{T}_{\text {ave }} \\
C_{2} \text { : It corresponds to period of operation when radar } \\
\text { time is critical. } \\
C_{2}=\bar{E}_{\text {ave }}+10^{5} \bar{T}_{\text {ave }} \\
\text { (A.39) } \\
\text { Where, } \bar{E}_{\text {ave }}=\text { Average energy per second; } \bar{T}_{\text {ave }}= \\
\text { Average radar time per second. }\end{array}\end{array}$ & [8] \\
\hline 4 & Average Power & $\begin{array}{l}\text { Rate of energy flow averaged over one full period. } \\
\qquad P_{\text {avg }}=\frac{\text { Pulse Width }(\tau)}{\operatorname{PRT}(T)} \cdot \text { Peak Power }\end{array}$ & [8], [27] \\
\hline
\end{tabular}

Tab. 1. Performance measures. 


\begin{tabular}{|c|c|c|c|c|c|c|c|c|c|c|c|c|c|c|c|c|}
\hline \multirow[t]{2}{*}{ SINo } & $\begin{array}{c}\text { Track Length } \\
\text { (s) }\end{array}$ & $\begin{array}{l}\text { Max. Acc. } \\
\left(\mathrm{m} / \mathrm{s}^{2}\right)\end{array}$ & $\begin{array}{c}\text { Man. Density } \\
(\%)\end{array}$ & $\begin{array}{l}\text { Number of } \\
\text { Waveforms }\end{array}$ & \multicolumn{2}{|c|}{$\begin{array}{l}\text { Ave.Power } \\
\text { (W) }\end{array}$} & \multicolumn{2}{|c|}{$\begin{array}{l}\text { Pos.RMSE } \\
(\mathrm{m})\end{array}$} & \multicolumn{2}{|c|}{$\begin{array}{l}\text { Vel.RMSE } \\
(\mathrm{m} / \mathrm{s})\end{array}$} & \multicolumn{2}{|c|}{$\begin{array}{l}\text { Cost } \\
C_{1}\end{array}$} & \multicolumn{2}{|c|}{$\begin{array}{c}\text { Cost } \\
C_{2}\end{array}$} & \multicolumn{2}{|c|}{$\begin{array}{c}\text { Track loss } \\
(\%)\end{array}$} \\
\hline & \multicolumn{16}{|c|}{ Benchmark Trajectory -1} \\
\hline 1 & \multirow{5}{*}{165} & \multirow{5}{*}{29.4} & \multirow{5}{*}{24.24} & 5 & 3.14 & & 184.26 & \multirow{5}{*}{$115^{1}$} & 97.64 & \multirow{5}{*}{$50.27^{1}$} & 3.61 & \multirow{5}{*}{$7.63^{1}$} & 50.15 & \multirow{5}{*}{$41.65^{1}$} & 0 & \multirow{5}{*}{$0^{1}$} \\
\hline 2 & & & & 10 & 2.85 & & 121.12 & & 90.58 & & 3.31 & & 48.53 & & 0 & \\
\hline 3 & & & & 20 & 2.60 & $7.28^{1}$ & 100.17 & & 85.15 & & 3.04 & & 47.31 & & 0 & \\
\hline 4 & & & & 40 & 2.55 & & 98.62 & & 84.31 & & 2.78 & & 46.89 & & 0 & \\
\hline 5 & & & & 50 & 2.52 & & 97.31 & & 84.31 & & 2.78 & & 46.83 & & 0 & \\
\hline \multicolumn{17}{|c|}{ Benchmark Trajectory -2} \\
\hline 6 & \multirow{5}{*}{150} & \multirow{5}{*}{39.2} & \multirow{5}{*}{34.66} & 5 & 3.09 & & 259.48 & \multirow{5}{*}{$100.3^{1}$} & 105.36 & \multirow{5}{*}{$52.8^{1}$} & 3.56 & & 50.52 & & 0 & \\
\hline 7 & & & & 10 & 3.42 & & 226.29 & & 104.51 & & 3.91 & & 52.03 & & 0 & \\
\hline 8 & & & & 20 & 2.96 & $6.16^{1}$ & 212.60 & & 94.24 & & 3.43 & $6.51^{1}$ & 49.77 & $40.84^{1}$ & 0 & $0^{1}$ \\
\hline 9 & & & & 40 & 2.88 & & 209.31 & & 95.28 & & 3.37 & & 48.64 & & 0 & \\
\hline 10 & & & & 50 & 2.79 & & 206.42 & & 89.65 & & 3.31 & & 48.59 & & 0 & \\
\hline & & & & & & nchmar & Trajector & & & & & & & & & \\
\hline 11 & & & & 5 & 7.34 & & 235.37 & & 130.26 & & 7.93 & & 66.13 & & 2 & \\
\hline 12 & & & & 10 & 7.16 & & 191.24 & & 134.79 & & 7.82 & & 66.02 & & 1.5 & \\
\hline 13 & 145 & 39.2 & 20.83 & 20 & 7.03 & $10.36^{1}$ & 187.41 & $148.7^{1}$ & 129.32 & $79.15^{1}$ & 7.78 & $10.71^{1}$ & 65.67 & $45.18^{1}$ & 1.5 & $0^{1}$ \\
\hline 14 & & & & 40 & 6.93 & & 178.63 & & 128.47 & & 7.32 & & 65.21 & & 1.5 & \\
\hline 15 & & & & 50 & 6.72 & & 177.38 & & 127.91 & & 7.01 & & 65.07 & & 1.5 & \\
\hline & & & & & & nchmarl & Trajector & & & & & & & & & \\
\hline 16 & & & & 5 & 7.29 & & 157.62 & & 66.31 & & 7.89 & & 65.72 & & 0.8 & \\
\hline 17 & & & & 10 & 7.19 & & 132.73 & & 64.28 & & 7.75 & & 65.61 & & 0 & \\
\hline 18 & 184 & 58.8 & 9.92 & 20 & 7.13 & $3.07^{1}$ & 103.28 & $45.81^{1}$ & 61.49 & $36.55^{1}$ & 7.71 & $3.42^{1}$ & 65.47 & $37.37^{1}$ & 0 & $0^{1}$ \\
\hline 19 & & & & 40 & 7.10 & & 99.86 & & 59.32 & & 7.68 & & 65.39 & & 0 & \\
\hline 20 & & & & 50 & 7.06 & & 99.27 & & 58.14 & & 7.63 & & 65.30 & & 0 & \\
\hline & & & & & & nchmarl & Trajector & & & & & & & & & \\
\hline 21 & & & & 5 & 7.41 & & 237.38 & & 107.61 & & 7.92 & & 66.01 & & 1.8 & \\
\hline 22 & & & & 10 & 7.30 & & 236.49 & & 105.74 & & 7.84 & & 65.92 & & 1.3 & \\
\hline 23 & 182 & 68.6 & 17.5 & 20 & 7.26 & $15.91^{1}$ & 210.71 & $171.5^{1}$ & 112.31 & $74.49^{1}$ & 7.79 & $16.27^{1}$ & 65.49 & $51.94^{1}$ & 0.8 & $0^{1}$ \\
\hline 24 & & & & 40 & 7.19 & & 206.46 & & 108.72 & & 7.74 & & 65.37 & & 0.8 & \\
\hline 25 & & & & 50 & 7.12 & & 202.13 & & 106.97 & & 7.68 & & 65.22 & & 0.8 & \\
\hline & & & & & & nchmar & Trajector & & & & & & & & & \\
\hline 26 & & & & 5 & 4.47 & & 201.28 & & 126.37 & & 4.78 & & 57 & & 2.3 & \\
\hline 27 & & & & 10 & 4.36 & & 193.79 & & 124.81 & & 4.63 & & 56.24 & & 2.1 & \\
\hline 28 & 188 & 68.6 & 18 & 20 & 4.29 & $7.62^{1}$ & 160.64 & $114.8^{1}$ & 122.29 & $72.44^{1}$ & 4.57 & $7.99^{1}$ & 56.08 & $44.48^{1}$ & 2.1 & $1^{1}$ \\
\hline 29 & & & & 40 & 4.16 & & 158.31 & & 120.76 & & 4.46 & & 55.91 & & 1.6 & \\
\hline 30 & & & & 50 & 4.01 & & 156.52 & & 118.62 & & 4.39 & & 55.71 & & 1.6 & \\
\hline
\end{tabular}

${ }^{1}$-Authors [10] ignored multipath effects.

Tab. 2. Comparison results for Benchmark Targets in the presence of SOJ+ Clutter + multipath + FA.

\begin{tabular}{|c|c|c|c|c|c|c|c|c|c|c|}
\hline SINo & $\begin{array}{l}\text { Track Length } \\
\text { (s) }\end{array}$ & $\begin{array}{l}\text { Max. Acc. } \\
\left(\mathrm{m} / \mathrm{s}^{2}\right)\end{array}$ & $\begin{array}{c}\text { Man. Density } \\
(\%)\end{array}$ & $\begin{array}{l}\text { Number of } \\
\text { Waveforms }\end{array}$ & $\begin{array}{l}\text { Ave.Power } \\
\text { (W) }\end{array}$ & $\begin{array}{l}\text { Pos.RMSE } \\
\text { (m) }\end{array}$ & $\begin{array}{c}\text { Vel.RMSE } \\
(\mathrm{m} / \mathrm{s})\end{array}$ & $\begin{array}{c}\text { Cost } \\
C_{1}\end{array}$ & $\begin{array}{c}\text { Cost } \\
C_{2}\end{array}$ & $\begin{array}{c}\text { Track loss } \\
(\%)\end{array}$ \\
\hline \multicolumn{11}{|c|}{ Benchmark Trajectory -1 } \\
\hline 1 & \multirow{5}{*}{165} & \multirow{5}{*}{29.4} & \multirow{5}{*}{24.24} & 5 & 3.03 & 185 & 98.47 & 3.49 & 49.47 & 0 \\
\hline 2 & & & & 10 & 2.71 & 139.66 & 88.21 & 3.16 & 47.65 & 0 \\
\hline 3 & & & & 20 & 2.63 & 123.28 & 83.94 & 3.08 & 47.45 & 0 \\
\hline 4 & & & & 40 & 2.58 & 117.62 & 82.61 & 2.93 & 46.28 & 0 \\
\hline 5 & & & & 50 & 2.51 & 116.31 & 79.42 & 2.82 & 46.21 & 0 \\
\hline \multicolumn{11}{|c|}{ Benchmark Trajectory -2} \\
\hline 6 & \multirow{5}{*}{150} & \multirow{5}{*}{39.2} & \multirow{5}{*}{34.66} & 5 & 3.13 & 257.74 & 105.98 & 3.61 & 50.62 & 0 \\
\hline 7 & & & & 10 & 3.06 & 249.07 & 106.53 & 3.53 & 50.28 & 0 \\
\hline 8 & & & & 20 & 3 & 246.85 & 103.35 & 3.47 & 49.98 & 0 \\
\hline 9 & & & & 40 & 2.98 & 242.61 & 102.15 & 3.36 & 47.32 & 0 \\
\hline 10 & & & & 50 & 2.73 & 239.37 & 101.61 & 3.32 & 45.23 & 0 \\
\hline \multicolumn{11}{|c|}{ Benchmark Trajectory -3} \\
\hline 11 & \multirow{5}{*}{145} & \multirow{5}{*}{39.2} & \multirow{5}{*}{20.83} & 5 & 7.43 & 229.67 & 147.94 & 8.76 & 68.01 & 2.6 \\
\hline 12 & & & & 10 & 7.39 & 225.36 & 144.45 & 8.41 & 67.82 & 2.4 \\
\hline 13 & & & & 20 & 7.35 & 216.13 & 138.27 & 8.32 & 67.39 & 2.0 \\
\hline 14 & & & & 40 & 7.16 & 199.62 & 132.69 & 7.83 & 66.97 & 1.7 \\
\hline 15 & & & & 50 & 7.12 & 198.79 & 130.71 & 7.78 & 66.83 & 1.7 \\
\hline \multicolumn{11}{|c|}{ Benchmark Trajectory -4} \\
\hline 16 & \multirow{5}{*}{184} & \multirow{5}{*}{58.8} & \multirow{5}{*}{9.92} & 5 & 7.32 & 144.38 & 69.86 & 7.92 & 65.87 & 1.2 \\
\hline 17 & & & & 10 & 7.28 & 138.92 & 69.37 & 7.86 & 65.70 & 0 \\
\hline 18 & & & & 20 & 7.21 & 129.76 & 68.81 & 7.74 & 65.59 & 0 \\
\hline 19 & & & & 40 & 7.07 & 120.89 & 67.43 & 7.43 & 65.06 & 0 \\
\hline 20 & & & & 50 & 7.04 & 118.67 & 66.94 & 7.39 & 64.93 & 0 \\
\hline \multicolumn{11}{|c|}{ Benchmark Trajectory -5} \\
\hline 21 & \multirow{5}{*}{182} & \multirow{5}{*}{68.6} & \multirow{5}{*}{17.5} & 5 & 7.32 & 226.17 & 128.39 & 7.91 & 66.03 & 0 \\
\hline 22 & & & & 10 & 7.26 & 215.48 & 125.73 & 7.88 & 65.95 & 0 \\
\hline 23 & & & & 20 & 7.19 & 213.62 & 124.54 & 7.76 & 65.82 & 0 \\
\hline 24 & & & & 40 & 7.09 & 209.36 & 123.89 & 7.69 & 65.62 & 0 \\
\hline 25 & & & & 50 & 7.03 & 208.97 & 123.28 & 7.42 & 65.54 & \\
\hline \multicolumn{11}{|c|}{ Benchmark Trajectory -6} \\
\hline 26 & \multirow{5}{*}{188} & \multirow{5}{*}{68.6} & \multirow{5}{*}{18} & 5 & 4.79 & 178.19 & 142.69 & 5.06 & 55.86 & 3.1 \\
\hline 27 & & & & 10 & 4.63 & 173.50 & 140.72 & 5.01 & 55.29 & 2.2 \\
\hline 28 & & & & 20 & 4.54 & 170.42 & 132.26 & 4.82 & 55.07 & 2.2 \\
\hline 29 & & & & 40 & 4.46 & 169.38 & 131.63 & 4.78 & 54.91 & 2.2 \\
\hline 30 & & & & 50 & 4.32 & 168.74 & 130.91 & 4.71 & 54.86 & 2.2 \\
\hline
\end{tabular}

Tab. 3. Comparison results for Benchmark Targets in the presence of FA + SSJ+ Clutter + Multipath. 